Running Head: TRIANGLES IN PACKAGING DESIGN

Resubmitted to: Food Quality and Preference (May 2019)

\title{
On the costs and benefits of using triangles in packaging design
}

Hui Zhao ${ }^{1}$, Yuxuan $\mathrm{Qi}^{1}$, Charles Spence ${ }^{2}, \&$ Xiaoang Wan ${ }^{1}$

1. Department of Psychology, Tsinghua University, Beijing, China

2. Crossmodal Research Laboratory, Department of Experimental Psychology, University of Oxford, $U K$

Correspondence: Xiaoang Wan, Department of Psychology, School of Social Sciences, Tsinghua University, Beijing, China 100084.

Email:wanxa@mail.tsinghua.edu.cn 


\begin{abstract}
A study was conducted to investigate the factors that influence the visual search for bottles displaying a triangular label whose orientation differs from that of background distractors, using shampoo bottles as stimuli. In Experiments 1 and 2, the participants were asked to find a bottle having a triangular label in a different orientation from the others displayed. The results consistently demonstrated that searching for a trapezoidal-shaped bottle with a downward-pointing triangular label was faster than searching for the same bottle with an upward-pointing triangular label instead. In Experiment 3, participants rated the valence and bottle-label congruency for all of the packaging exemplars used in Experiments 1 and 2. The results revealed that the participants rated a downward-pointing triangular-shaped label to be more incongruent with trapezoidal-shaped bottles than an upward-pointing label. Our findings reveal that the downward-pointing triangle superiority (DPTS) effect elicited by the triangular-shaped label is also influenced by the silhouette of the bottle as a reference frame. Such results have direct implications for packaging design.
\end{abstract}

Key words: visual search; design; label; triangle; packaging 


\section{Introduction}

Purchasing decisions are often influenced by the interaction between attention, working memory, and preferences (e.g., Orquin \& Loose, 2013). As such, capturing the consumers' attention in-store (or increasingly online) might increase the likelihood of the products being purchased (Pieters \& Warlop, 1999; Pieters, Warlop, \& Wedel, 2002; Spence, 2016; Towal, Mormann, \& Koch, 2013). Given that consumers typically spend very little time when making their purchasing decisions (Zbib, Wooldridge, Ahmed, \& Benlian, 2010), simple striking visual features, such as the design elements of product packaging, might well be expected to play a crucial role in this type of rapid decision-making (Clement, Kristensen, \& Grønhaug, 2013; Simmonds \& Spence, 2017; Wang, 2013). As an integral part of the processes of purchase and consumption, product packaging has become an important marketing tool (Grundey, 2010; Selame \& Koukos, 2002). Packaging is often designed to appeal visually to attract the attention of the consumer. Successful packaging increases the hedonic value of the products, and/or supports brand positioning (Chitturi, Raghunathan, \& Mahajan, 2008; Creusen \& Schoormans, 2005; Orth \& Malkewitz, 2008; Pieters, Wedel, \& Batra, 2010; Stoll, Baecke, \& Kenning, 2008).

Recently, a series of laboratory studies have demonstrated that people find a wine bottle with a downward-pointing triangle on its label more rapidly than when searching for an identical bottle with an upward-pointing triangle instead (Zhao, Huang, Spence, \& Wan, 2017; Zhao, Spence, \& Wan, 2016). Such an effect for wine labels is based on laboratory-based research on the visual search for simple geometric 
shapes showing that the search for a downward-pointing triangle among upward-pointing distractor triangles is faster than vice versa (Larson, Aronoff, \& Stearns, 2007). This is known as the downward-pointing triangle superiority (DPTS) effect (Shen, Wan, Mu, \& Spence, 2015).

The emergence of the DPTS effect for simple geometric shapes might be attributed to the threat-related information conveyed by a downward-pointing triangle, presumably because it resembles an angry face with muscles and eyes pulling down to form a "V' shape (Larson, Aronoff, \& Steuer, 2012; Salgado-Montejo, Salgado, Alvarado, \& Spence, 2017). If downward-pointing triangles were to elicit the processing of threat-related information (Larson, Aronoff, Sarinopoulos, \& Zhu, 2009), then it should come as no surprise to find that downward-pointing triangles capture visual attention more readily than neutral stimuli such as upward-pointing triangles (Larson et al., 2007, 2012; Watson, Blagrove, Evans, \& Moore, 2012). However, this threat-related explanation of the DPTS effect may not easily be used to account for the results documented with non-threatening images of triangular-shaped foods and pizza packaging (Shen et al., 2015) or wine bottles (Zhao et al., 2016, 2017). Moreover, Velasco and colleagues (2015) also found that participants preferred the triangular-shaped label shown on a wine label to point downward. Alternatively, the DPTS effect might also be explained by the possibility that downward-pointing triangles have certain perceptual features, such as a lack of stability (Shen et al., 2015), or perhaps being incongruent with the surrounding frame (Zhao et al., 2017). For example, a wine bottle often has an upward-pointing silhouette due to the smaller 
width of its neck than of its body, so placing a downward-pointing triangular-shaped label on a wine bottle might be analogous to presenting an element in an orientation differing from that of the reference frame (see Kunde \& Hoffman, 2000; May \& Li, 2009). Considering that searching for an oriented feature is influenced by the surrounding frame (see Marendaz, 1998, for a review), it would seem reasonable to expect that the DPTS effect elicited by the orientation of the triangular-shaped label would be diminished, or even eliminated, if the contour of packaging happens not to have an upward-pointing form.

In order to test this possibility, we examined the DPTS effects with triangular-shaped labels attached to product packaging with more varied shapes. We chose shampoo bottles as our experimental stimuli, given that shampoo is often packaged in plastic bottles whose design elements include (1) the size, shape, colour, and material (feel) of the bottle, (2) the type and location of the bottle cap, and (3) distinctive label(s). In our observation, the downward-pointing triangular shapes have been used for packaging of personal care products, such as the "Perfectslim" product line of L'Oreal (see Figure 1 for illustrations of these popular commercial products). The silhouette of a bottle can help consumers recognize product category (Arboleda \& Arce-Lopera, 2015), and the design of shampoo bottles is also associated with a distinctive brand identity (Zaichkowsky, 2010).

INSERT FIGURE 1 ABOUT HERE 
In order to create congruence or incongruence between the label and bottle silhouette, three different types of bottle silhouettes were chosen for study (see Figure 2 for illustrations of our experimental stimuli), including an inverted trapezoidal-shaped bottle which has a downward-pointing $\mathrm{V}$ form in its contour (i.e., the bottom was $23.5 \%$ narrower than the top), a trapezoidal-shaped bottle with an upward-pointing $\mathrm{V}$ form in its contour (i.e., the top was $23.5 \%$ narrower than the bottom), and a rectangular-shaped bottle in which the top and bottom were of the same widths. The latter included as a baseline control condition.

\section{INSERT FIGURE 2 ABOUT HERE}

If the influence of a triangular element on visual search for bottles is solely attributable to its own orientation, then one would expect to observe significant DPTS effects for all three bottle silhouettes. Alternatively, however, it is also possible that bottle silhouette moderates the DPTS effect elicited by triangular labels, and it might be that the incongruence between the orientation of label and bottle silhouette plays a more important role than the orientation of label itself. If this were to be true, one would expect to observe a significant DPTS effect with the trapezoidal-shaped bottle, presumably due to the incongruence between a downward-pointing label and bottle silhouette). Moreover, one would also expect to observe a reversed DPTS effect with the inverted trapezoidal-shaped bottle due to the incongruence between an upward-pointing label and the bottle silhouette), and no significant difference between 
TRIANGLES IN PACKAGING DESIGN

two types of labels for rectangular-shaped bottles. The findings of the present study would therefore reveal the costs and benefits of using a triangular shape as a design element in packaging design, in terms of capturing consumers' attention and influencing their evaluations of the products. Drawing on the literature on the reference frame, the findings of the present study would also provide empirical evidence regarding how two important factors to consider in packaging design, i.e., graphic design element and packaging shape, interact to influence consumers' detection and evaluations of the products.

\section{Experiment 1}

Methods

\section{Participants}

Sixty participants (mean age $=20.98 \pm 2.08$ years; 31 females) from mainland China took part in the present study. They were randomly divided into three groups, with the constraint that each group had approximately equal numbers of males and females. Therefore, we used the same sample size for each group $(\mathrm{N}=20)$ as in Zhao et al.'s (2016) previous study.

In this and the following experiments, all of the participants reported that they were right-handed, that they had normal or corrected-to-normal vision (including normal colour vision). The present research was approved by the ethics committee of the Psychology Department of Tsinghua University, and performed in accordance with the ethical standards laid down in the Declaration of Helsinki. In this and the following experiments, each participant gave their written informed consent at the beginning of the study, and was monetarily compensated for their time and 
participation.

\section{Apparatus and stimuli}

The experiment was conducted on Pentium-based computers, with 16-inch screens set to $1024 \times 768$ pixels with a refresh rate of $60 \mathrm{~Hz}$. The software MATLAB $2013 \mathrm{~b}$ installed with PsychToolbox 3.0 was used to present the stimuli and to record the data. The participants were instructed to sit approximately $50 \mathrm{~cm}$ from the screen. All of the images presented were edited via the Adobe Photoshop software.

Each display (subtending $27.65^{\circ}$ horizontally x $27.65^{\circ}$ vertically) consisted of 16 bottles. Each bottle $\left(2.15^{\circ}\right.$ horizontally $\times 5.72^{\circ}$ vertically $)$ was placed at the centre of each cell of an invisible $4 \times 4$ matrix $\left(6.57^{\circ} \times 6.57^{\circ}\right)$ centred on the screen. As shown in Figure 3, three different types of bottle silhouettes were used. These included an inverted trapezoidal-shaped bottle (Bottle 1A), a trapezoidal-shaped bottle (Bottle 1B), and a rectangular-shaped bottle in which the top and bottom were of the same width (Bottle 1C). Each bottle had a silvery-grey flip-top cap on top of a green body (R: 140, G: 177, B: 95$)$, with a black triangular-shaped design element $\left(1.09^{\circ}\right.$ horizontally $\times$ $0.93^{\circ}$ vertically) pointing either downward or upward.

\section{INSERT FIGURE 3 ABOUT HERE}

\section{Design and procedure}

Based on the orientation of the triangular labels, all of the displays were classified as either oddball-present displays in which one label had a different orientation from 
the others (i.e., one downward-pointing triangle amongst fifteen upward-pointing triangles or vice versa), or oddball-absent displays in which all of the labels pointed in the same direction. For the oddball-present trials, we used a 2 (Label Orientation: downward- or upward-pointing label) $\times 3$ (Bottle Silhouette: inverted trapezoid, trapezoid, or rectangle) mixed-design, with Label Orientation as a within-participants factor and Bottle Silhouette being the between-participants factor. In other words, each participant was only shown one type of bottle silhouette, and all of the bottles within each display had the same shape. Similarly, we used an orthogonal design of Stimulus Orientation $\times$ Bottle Silhouette mixed-design for oddball-absent trials. However, we only focused on the oddball-present trials in the data analyses.

After finishing a practice block consisting of 20 trials, each participant completed 4 blocks of 64 trials each. Within each block, equal numbers of oddball-present and oddball-absent trials of different types were mixed and presented in a random order. At the beginning of each trail, a blank screen with a centred fixation (subtending $0.73^{\circ}$ horizontally $\times 0.73^{\circ}$ vertically) was presented for $800 \mathrm{~ms}$, followed by a search display which was presented until the participant responded. The participants were instructed to press one of two keys on the keyboard as rapidly and accurately as possible, in order to indicate whether all the bottles on each display were the same or not. The intertrial interval was set at $2 \mathrm{~s}$, and a beep sound was played over the participant's headphones to alert them of any incorrect response.

Results 
Overall, the participants were highly accurate $\left(\mathrm{M}_{\mathrm{acc}}=95.5 \%, \mathrm{SD}_{\mathrm{acc}}=3.1 \%\right)$ in their responses. We excluded those trials in which the RTs were shorter than $150 \mathrm{~ms}$ or longer than two standard deviations beyond the group mean from the following data analyses, resulting in $4.0 \%$ of the data being discarded. Mean accuracy and RTs calculated based on correct trials in each condition of oddball-present trials are also shown in Figure 3.

The 2 (Label Orientation: downward- or upward-pointing) $\times 3$ (Bottle Silhouette: inverted trapezoid, trapezoid, or rectangle) mixed-design Analyses of Variance (ANOVAs) were performed on the RT and accuracy data from the oddball-present trials. The results revealed a significant main effect of Label Orientation on the accuracy data, $F(1,57)=7.20, p=0.01, \eta_{\mathrm{p}}{ }^{2}=0.11$, suggesting that searching for a downward-pointing triangular label was less accurate overall $\left(\mathrm{M}_{\mathrm{acc}}=91.9 \%, \mathrm{SD}_{\mathrm{acc}}=\right.$ $6.0 \%)$ than searching for an upward-pointing triangular label $\left(\mathrm{M}_{\mathrm{RT}}=93.6 \%, \mathrm{SD}_{\mathrm{RT}}=\right.$ $5.1 \%)$. The results also revealed a significant interaction term on the $\mathrm{RTs}, F(2,57)=$ 4.22, $p=0.02, \eta_{\mathrm{p}}{ }^{2}=0.13$. None of other main or interaction effects was significant on the RT or accuracy data, all $F_{\mathrm{S}}<1.89, p \mathrm{~s}>0.16$.

In order to interpret the interaction term on the RTs, pairwise comparisons were performed for each type of bottle silhouette. As for Bottle 1B, searching for a downward-pointing triangular label $\left(\mathrm{M}_{\mathrm{RT}}=1742 \mathrm{~ms}, \mathrm{SD}_{\mathrm{RT}}=231 \mathrm{~ms}\right)$ was faster than searching for an upward-pointing triangular label $\left(\mathrm{M}_{\mathrm{RT}}=1803 \mathrm{~ms}, \mathrm{SD}_{\mathrm{RT}}=206 \mathrm{~ms}\right)$, $t(19)=2.42, p=0.03$, Cohen's $d=0.55$, indicating a significant DPTS effect of $61 \mathrm{~ms}$. By contrast, the RTs of searching for the downward- and upward-pointing targets 
were comparable for Bottle $1 \mathrm{~A}$ or Bottle $1 \mathrm{C}$, both $t \mathrm{~s}<1.51, p \mathrm{~s}>0.14$. Analogous analyses on the accuracy data revealed that searching for a downward-pointing triangular target $\left(\mathrm{M}_{\mathrm{acc}}=90.5 \%, \mathrm{SD}_{\mathrm{acc}}=6.2 \%\right)$ was less accurate than searching for an upward-pointing triangular target $\left(\mathrm{M}_{\mathrm{acc}}=93.2 \%, \mathrm{SD}_{\mathrm{acc}}=6.1 \%\right)$ for Bottle $1 \mathrm{~A}, t(19)=$ 2.22, $p=.04$, Cohen's $d=0.51$, suggesting a reversed DPTS pattern; whereas no such effect was significant for Bottles $1 \mathrm{~B}$ and $1 \mathrm{C}$, both $t \mathrm{~s}<1.60, p \mathrm{~s}>0.12$.

Discussion

In summary, the results of Experiment 1 revealed a significant DPTS effect in participants' visual search for a trapezoidal-shaped shampoo bottle having a triangular-shaped label whose orientation was different from the rest of the display items. Considering such a bottle also has an upward-pointing (inverted V) form in its contour, this result is consistent with Zhao et al.'s $(2016,2017)$ recent findings with wine bottles. By contrast, a trend towards a reversed DPTS effect was observed in the accuracy data for the inverted trapezoidal-shaped bottle. Considering that the inverted trapezoidal-shaped bottle has a downward-pointing $\mathrm{V}$ form in its contour, the higher accuracy rates documented for an upward-pointing triangular label for such a bottle might be also interpreted by the incongruence in orientation of bottle silhouette and label, whereas the absence of a reversed DPTS effect in the RTs for the inverted trapezoidal-shaped bottle may be due to a speed-accuracy trade-off in the data. Moreover, no DPTS or reversed DPTS effect was observed with the bottle having a rectangular shape.

Collectively, these results are in line with the prediction of the account that the 
incongruence between the orientation of label and bottle silhouette influences the participants' visual search, thus suggesting that bottle silhouette might function as a reference frame to constraint the DPTS effect elicited by the triangular label. However, it should be noted that it is not unusual for personal care products to have their flip-top caps on the bottom of the packaging to facilitate dispensing. Therefore, in the next experiment, we further tested the DPTS effect with bottles having the same design as those in Experiment 1 but with their caps on the bottom in Experiment 2.

\section{Experiment 2}

Methods

Sixty new participants (mean age $=20.88 \pm 1.98$ years; 30 females) took part in Experiment 2, none of whom had taken part in the previous experiment. Once again, the participants were randomly divided into three groups. All aspects of the present study were the same as those in Experiment 1 except for the stimuli being used. As shown in Figure 4, we presented Bottles 2A-C which had the same design as Bottles 1A-C, respectively, but with the silver flip-top caps now on the bottom.

\section{INSERT FIGURE 4 ABOUT HERE}

Results

Overall, the participants were highly accurate $\left(\mathrm{M}_{\mathrm{acc}}=94.9 \%, \mathrm{SD}_{\mathrm{acc}}=4.1 \%\right)$ in their responses. We excluded RTs shorter than $150 \mathrm{~ms}$ or longer than two standard deviations beyond the group mean from our data analyses, resulting in the discarding 
of $3.9 \%$ of the data. Mean accuracy and RTs for correct trials in each condition of oddball-present trials are shown in Figure 4.

The Label Orientation $\times$ Bottle Silhouette ANOVAs on the oddball-present trials revealed a marginally significant main effect of Label Orientation on the RTs, $F(1,57)$ $=3.71, p=0.059, \eta_{\mathrm{p}}^{2}=0.06$, but it was qualified by a significant interaction term on the RTs, $F(2,57)=6.85, p<0.01, \eta_{\mathrm{p}}^{2}=0.19$. Such an interaction term was also significant for the accuracy data, $F(2,57)=5.98, p<0.01, \eta_{\mathrm{p}}^{2}=0.17$, whereas none of other effects was significant, all $F_{\mathrm{s}}<1.72, p \mathrm{~s}>0.19$. Pairwise comparisons revealed a significant DPTS effect of $72 \mathrm{~ms}$ for Bottle $2 \mathrm{~B}$, as searching for a downward-pointing triangular label $\left(\mathrm{M}_{\mathrm{RT}}=1574 \mathrm{~ms}, \mathrm{SD}_{\mathrm{RT}}=412 \mathrm{~ms}\right)$ was faster than for an upward-pointing one $\left(\mathrm{M}_{\mathrm{RT}}=1646 \mathrm{~ms}, \mathrm{SD}_{\mathrm{RT}}=404 \mathrm{~ms}\right), t(19)=3.23, p<0.01$, Cohen's $d=0.72$. By contrast, the RTs of searching for the downward- and upward-pointing targets were comparable for Bottle $2 \mathrm{~A}$ and Bottle $2 \mathrm{C}$, both $t \mathrm{~s}<1.25$, $p \mathrm{~s}>0.22$. Analogous analyses on the accuracy data revealed that searching for a downward-pointing triangular label $\left(\mathrm{M}_{\mathrm{acc}}=88.1 \%, \mathrm{SD}_{\mathrm{acc}}=9.2 \%\right)$ was less accurate than for an upward-pointing one $\left(\mathrm{M}_{\mathrm{acc}}=91.0 \%, \mathrm{SD}_{\mathrm{acc}}=7.8 \%\right)$ for Bottle $2 \mathrm{~A}, t(19)=$ 3.01, $p<0.01$, Cohen's $d=0.70$. Similarly, searching for a downward-pointing triangular label was slightly more accurate $\left(\mathrm{M}_{\mathrm{acc}}=93.9 \%, \mathrm{SD}_{\mathrm{acc}}=6.2 \%\right)$ than for an upward-pointing one $\left(\mathrm{M}_{\mathrm{acc}}=91.8 \%, \mathrm{SD}_{\mathrm{acc}}=7.5 \%\right)$ for Bottle $2 \mathrm{C}, t(19)=1.93, p=$ 0.069, Cohen's $d=0.44$. By contrast, no such effect was significant for Bottle $2 \mathrm{~B}$, $t(19)=1.48, p=0.16$.

Next, the data from Experiments 1 and 2 were combined, and 2 (Label 
Orientation: downward- or upward-pointing) $\times 3$ (Bottle Silhouette: inverted trapezoid, trapezoid, or rectangle) $\times 2$ (Cap Location: top or bottom) mixed-design ANOVAs were performed on the RT and accuracy data of the oddball-present trials. Label Orientation was treated as a within-participants factor, whereas Bottle Silhouette and Cap Location were treated as between-participants factors. The main effect of Label Orientation was significant for the accuracy data, $F(1,114)=8.07, p=$ $0.005, \eta_{\mathrm{p}}{ }^{2}=0.07$, and marginally significant for the RT data, $F(1,114)=3.44, p=$ $0.07, \eta_{\mathrm{p}}{ }^{2}=0.03$. However, both of these main effects were qualified by a significant Label Orientation $\times$ Bottle Silhouette interaction, RTs: $F(2,114)=10.52, p<0.001$, $\eta_{\mathrm{p}}{ }^{2}=0.16$, and accuracy: $F(2,114)=3.98, p=0.02, \eta_{\mathrm{p}}{ }^{2}=0.07$. None of other main or interaction effects was significant, all $F \mathrm{~s}<2.44, p \mathrm{~s}>0.09$.

Pairwise comparisons on the combined data of Experiments 1 and 2 revealed that searching for a downward-pointing triangular label $\left(\mathrm{M}_{\mathrm{RT}}=1658, \mathrm{SD}_{\mathrm{RT}}=340 \mathrm{~ms}\right)$ was faster than for an upward-pointing one $\left(\mathrm{M}_{\mathrm{RT}}=1725, \mathrm{SD}_{\mathrm{RT}}=326 \mathrm{~ms}\right)$ for the trapezoidal-shaped bottles, $t(39)=4.00, p<0.001$, Cohen's $d=0.64$, indicative of a significant DPTS effect of $67 \mathrm{~ms}$. As for the inverted trapezoidal-shaped bottles, searching for a downward-pointing triangular label was slightly slower $\left(\mathrm{M}_{\mathrm{RT}}=1713\right.$, $\left.\mathrm{SD}_{\mathrm{RT}}=291 \mathrm{~ms}\right)$ and less accurate $\left(\mathrm{M}_{\mathrm{acc}}=89.3 \%, \mathrm{SD}_{\mathrm{acc}}=7.8 \%\right)$ than searching for an upward-pointing label $\left(\mathrm{M}_{\mathrm{RT}}=1686 \mathrm{~ms}, \mathrm{SD}_{\mathrm{RT}}=306 \mathrm{~ms}, \mathrm{M}_{\mathrm{acc}}=92.1 \%, \mathrm{SD}_{\mathrm{acc}}=7.0 \%\right)$, RTs: $t(39)=1.97, p=0.056$, Cohen's $d=0.32$, accuracy: $t(39)=3.66, p=0.001$, Cohen's $d=0.59$. As for the rectangular-shaped bottles, searching for the downwardand upward-pointing targets were comparable in terms of both RTs and accuracy, both 
$t \mathrm{~s}<0.54, p \mathrm{~s}>0.59$.

Discussion

Once again, the results of Experiment 2 revealed a significant DPTS effect for the trapezoidal-shaped bottle, consistent with our observation with the trapezoidal-shaped bottle in Experiment 1. We also observed a reversed DPTS trend in the accuracy data of the inverted trapezoidal-shaped bottle, whereas no DPTS effect was observed with the rectangular-shaped bottle. These results are also consistent with what we found with the inverted trapezoidal- and rectangular-shaped bottles in Experiment 1. When the data of Experiments 1 and 2 were combined, we even found a reversed DPTS trend in the RT data of the inverted trapezoidal-shaped bottles. Collectively, these results would therefore appear to suggest that bottle silhouette moderates the DPTS effect elicited by the triangular label, by functioning as a reference frame (e.g., Marendaz, 1998). The incongruence between the bottle contour and the orientation of the label might be a key factor to influence the participants' visual search.

When we analyzed the combined the data from Experiments 1 and 2, neither the main effect of Cap Location nor its interactions with any other factor was significant. Collectively, these results would therefore appear to suggest that simply moving the flip-top cap from the top (as in Experiment 1) to the bottom of the packaging (as in Experiment 2) exerted no influence on the overall RTs to detect the target or the DPTS effect. In Experiment 3, we further examined the subjective ratings of the bottles used in Experiments 1 and 2 in order to assess whether the participants were sensitive to the incongruence between the bottle contour and the orientation of the label. 


\section{Experiment 3}

In Experiment 3, we examined the subjective ratings of the different bottles used in Experiments 1 and 2. Specifically, we wanted to examine whether the perceived valence of the packaging would be influenced by the orientation of the triangular-shaped labels, bottle silhouette, and/or cap location. We also wanted to examine whether these three features interacted to influence the perceived incongruence of the object.

Methods

Forty new participants (mean age $=20.95 \pm 2.16$ years; 20 females) took part in this experiment, none of whom had taken part in either of the previous experiments. The experiment was conducted online at www.qualtrics.com. We used a 2 (Label Orientation: downward- or upward-pointing label) $\times 3$ (Bottle Silhouette: inverted trapezoid, trapezoid, or rectangle) $\times 2$ (Cap Location: top or bottom) within-participants design. Images of the 12 stimuli used in Experiments 1 and 2 were presented in a random order, one at a time. The participants were asked to complete the following rating tasks, both on 7-point scales. They first rated each image for its emotional valence (ranging from very unpleasant to very pleasant), and then rated to which extent a bottle and its label were congruent, with greater values indicating a greater degree of bottle-label congruency.

Results

The mean ratings scores for each bottle are shown in Figure 5. 


\section{INSERT FIGURE 5 ABOUT HERE}

\section{Valence ratings}

We first performed 2 (Label Orientation: downward- or upward-pointing) $\times 3$ (Bottle Silhouette: inverted trapezoid, trapezoid, or rectangle) $\times 2$ (Cap Location: top or bottom) repeated-measure ANOVAs on the valence scores. The results revealed a significant main effect of Label Orientation, $F(1,39)=7.79, p=0.008, \eta_{\mathrm{p}}{ }^{2}=0.17$, thus suggesting that bottles having downward-pointing triangular-shaped labels were considered to be less pleasant than those with labels pointing upwards $\left(\mathrm{M}_{\text {valence }}=4.0\right.$, $\mathrm{SD}_{\text {valence }}=0.7$ vs. $\mathrm{M}_{\text {valence }}=4.3, \mathrm{SD}_{\text {valence }}=0.6$ ). The results also revealed a significant main effect of Bottle Silhouette, $F(2,78)=18.16, p<0.001, \eta_{\mathrm{p}}{ }^{2}=0.32$, but it was qualified by a significant interaction term between Bottle Silhouette and Cap Location, $F(2,78)=17.98, p<0.001, \eta_{\mathrm{p}}{ }^{2}=0.32$. None of other main or interaction effects was significant, all $F \mathrm{~s}<2.67, p \mathrm{~s}>0.11$.

In order to interpret the significant interaction term between Bottle Silhouette and Cap Location, one-way repeated-measures ANOVAs were performed for the data of Bottles 1A, 1B, and 1C. The results revealed a significant main effect of Bottle Silhouette, $F(2,78)=26.47, p<0.001, \eta_{\mathrm{p}}{ }^{2}=0.40$. Pairwise comparisons with Bonferroni correction revealed that Bottle 1B $\left(\mathrm{M}_{\text {valence }}=3.4, \mathrm{SD}_{\text {valence }}=1.0\right)$ was considered to be less pleasant than Bottles $1 \mathrm{~A}\left(\mathrm{M}_{\text {valence }}=4.6, \mathrm{SD}_{\text {valence }}=1.0\right)$ and $1 \mathrm{C}$ $\left(\mathrm{M}_{\text {valence }}=4.7, \mathrm{SD}_{\text {valence }}=0.8\right)$, both $t \mathrm{~s}>4.95, p \mathrm{~s}<0.001$, whereas Bottles $1 \mathrm{~A}$ and $1 \mathrm{C}$ 
were considered as being equally pleasant, $t(39)=0.92, p>0.99$. By contrast, the main effect of Bottle Silhouette was marginally significant for Bottles 2A, 2B, and 2C, $F(2,78)=2.88, p=0.062, \eta_{\mathrm{p}}^{2}=0.07$. Pairwise comparisons with Bonferroni correction revealed that the difference between Bottle $2 \mathrm{~A}\left(\mathrm{M}_{\text {valence }}=3.8, \mathrm{SD}_{\text {valence }}=\right.$ $0.8)$ and Bottle $2 \mathrm{C}\left(\mathrm{M}_{\text {valence }}=4.2, \mathrm{SD}_{\text {valence }}=1.1\right)$ was marginally significant, $t(39)=$ 2.39, $p=0.066$, Cohen's $d=0.44$; whereas neither of the differences between Bottle $2 \mathrm{~B}\left(\mathrm{M}_{\text {valence }}=4.0, \mathrm{SD}_{\text {valence }}=0.7\right)$ and the other two bottles reached significance, both $t \mathrm{~s}<1.44, p \mathrm{~s}>0.48$.

Planned pairwise comparisons revealed that Bottle 1A with a downward-pointing label was considered to be less pleasant than the same bottle with an upward-pointing label $\left(\mathrm{M}_{\text {valence }}=4.4, \mathrm{SD}_{\text {valence }}=1.3\right.$ vs. $\left.\mathrm{M}_{\text {valence }}=4.8, \mathrm{SD}_{\text {valence }}=1.0\right), t(39)=2.43, p=$ 0.02, Cohen's $d=0.37$. A similar trend was also found for Bottle $2 \mathrm{C}\left(\mathrm{M}_{\text {valence }}=3.9\right.$, $\mathrm{SD}_{\text {valence }}=1.4$ vs. $\left.\mathrm{M}_{\text {valence }}=4.5, \mathrm{SD}_{\text {valence }}=1.1\right), t(39)=3.67, p=0.001$, Cohen's $d=$ 0.53 , but not for any other bottles, all $t \mathrm{~s}<1.15, p \mathrm{~s}>0.25$.

\section{Congruency ratings}

A Label Orientation $\times$ Bottle Silhouette $\times$ Cap Location ANOVA on the congruency scores revealed a significant main effect of Label Orientation, $F(1,39)=$ $11.08, p=0.002, \eta_{\mathrm{p}}^{2}=0.22$. This main effect was qualified by a significant interaction between Bottle Silhouette and Label Orientation, $F(2,78)=7.91, p=0.001, \eta_{\mathrm{p}}{ }^{2}=$ 0.17, and a significant interaction between Cap Location and Label Orientation, $F(2$, 78) $=6.97, p=0.012, \eta_{\mathrm{p}}^{2}=0.15$. The interaction term between Bottle Silhouette and Cap Location was also significant on the congruency ratings, $F(2,78)=11.16, p<$ 
$0.001, \eta_{\mathrm{p}}{ }^{2}=0.22$, whereas none of other effects was significant, all $F_{\mathrm{S}}<0.77, p \mathrm{~s}>$ 0.46 .

In order to interpret the significant Label Orientation $\times$ Bottle Silhouette interaction, the data for bottles with the same silhouette were combined and one-way repeated-measures ANOVAs were conducted. The results revealed a significant main effect of Label Orientation for the trapezoidal-shaped bottles (Bottles 1B and 2B), $F(1$, $39)=24.93, p<0.001, \eta_{\mathrm{p}}{ }^{2}=0.39$, and the rectangular-shaped bottles (Bottle 1C and 2C), $F(1,39)=5.24 p=0.028, \eta_{\mathrm{p}}{ }^{2}=0.12$. That is, compared to an upward-pointing label, a downward-pointing triangular-shaped label was considered as being more incongruent with the trapezoidal-shaped $\left(\mathrm{M}_{\text {congruency }}=3.4, \mathrm{SD}_{\text {congruency }}=0.9\right.$ vs. $\left.\mathrm{M}_{\text {congruency }}=4.4, \mathrm{SD}_{\text {congruency }}=1.2\right)$ or rectangular-shaped $\left(\mathrm{M}_{\text {congruency }}=3.8, \mathrm{SD}_{\text {congruency }}\right.$ $=1.0$ vs. $\mathrm{M}_{\text {congruency }}=4.3, \mathrm{SD}_{\text {congruency }}=1.3$ ) bottles. By contrast, no such effect was found for inverted trapezoidal-shaped bottles $\left(\mathrm{M}_{\text {congruency }}=4.0, \mathrm{SD}_{\text {congruency }}=1.2 \mathrm{vs}\right.$. $\left.\mathrm{M}_{\text {congruency }}=3.7, \mathrm{SD}_{\text {congruency }}=1.2\right), F(1,39)=0.88, p=0.35$.

In order to interpret the significant interaction terms between Label Orientation and Cap Location, we combined the data for bottles with the same design in cap location and conducted one-way repeated-measures ANOVAs. The results revealed a significant main effect of Label Orientation for those bottles with their caps on the top, $F(1,39)=17.38, p<0.001, \eta_{\mathrm{p}}^{2}=0.31$, thus suggesting that a downward-pointing label was considered to be less congruent with these bottles than an upward-pointing one $\left(\mathrm{M}_{\text {congruency }}=3.5, \mathrm{SD}_{\text {congruency }}=0.8\right.$ vs. $\left.\mathrm{M}_{\text {congruency }}=4.4, \mathrm{SD}_{\text {congruency }}=1.1\right)$. By contrast, no such difference was found for bottles with their caps on the bottom, $F(1$, 
$39)=0.02, p>0.89$, thus suggesting that downward- and upward-pointing labels were considered to be equally congruent with these bottles $\left(\mathrm{M}_{\text {congruency }}=3.9, \mathrm{SD}_{\text {congruency }}=\right.$ 1.1 vs. $\left.\mathrm{M}_{\text {congruency }}=3.9, \mathrm{SD}_{\text {congruency }}=1.0\right)$.

Planned pairwise comparisons revealed that, compared to an upward-pointing label, a downward-pointing label was considered to be less congruent with Bottle 1B $\left(\mathrm{M}_{\text {congruency }}=2.8, \mathrm{SD}_{\text {congruency }}=1.3\right.$ vs. $\left.\mathrm{M}_{\text {congruency }}=4.2, \mathrm{SD}_{\text {congruency }}=1.7\right), t(39)=4.62$, $p<0.001$, Cohen's $d=0.75$, Bottle $1 \mathrm{C}\left(\mathrm{M}_{\text {congruency }}=3.7, \mathrm{SD}_{\text {congruency }}=1.5\right.$ vs. $\left.\mathrm{M}_{\text {congruency }}=4.5, \mathrm{SD}_{\text {congruency }}=1.6\right), t(39)=2.54, p=0.015$, Cohen's $d=0.37$, and Bottle $2 \mathrm{~B}\left(\mathrm{M}_{\text {congruency }}=3.9, \mathrm{SD}_{\text {congruency }}=1.6\right.$ vs. $\left.\mathrm{M}_{\text {congruency }}=4.5, \mathrm{SD}_{\text {congruency }}=1.4\right)$, $t(39)=2.12, p=0.041$, Cohen's $d=0.33$. The opposite trend was observed for Bottle $2 \mathrm{~A}\left(\mathrm{M}_{\text {congruency }}=3.8, \mathrm{SD}_{\text {congruency }}=1.6\right.$ vs. $\left.\mathrm{M}_{\text {congruency }}=3.1, \mathrm{SD}_{\text {congruency }}=1.5\right), t(39)=$ 2.24, $p=0.031$, Cohen's $d=0.35$, but no significant results were found for Bottle $1 \mathrm{~A}$ or Bottle $2 \mathrm{C}$, both $t \mathrm{~s}<0.80, p \mathrm{~s}>0.43$.

Discussion

In summary, two major results emerge from the analysis of Experiment 3. First, a downward-pointing triangular-shaped label was considered to be more incongruent with the trapezoidal-shaped bottles, whereas these bottles were not considered as being more unpleasant when they had a downward-pointing label than when they had an upward-pointing label. Collectively, these results revealed that the DPTS effects documented with the trapezoidal-shaped bottles in Experiments 1 and 2, respectively, were more likely to be due to the incongruence between bottle contour and label orientation, rather than attributable solely to the label orientation itself. 
Second, the results of the present experiment also revealed that the participants considered bottles having downward-pointing triangular-shaped labels more unpleasant than those with labels pointing up overall. This result is in line with Westerman et al.'s (2013) findings with bottles of water or vodka, but contrast with Zhao et al.'s (2017) recent findings with bottles of wine (also see Velasco et al., 2015). The discrepancy between these studies suggests, at least tentatively, that the subjective ratings of the bottles with triangles might be modulated by the product category. That being said, our results also revealed that the influence of label orientation on the participants' valence ratings was constrained by other design elements, such as bottle silhouette and cap location. That is, both bottle silhouette and cap location influenced, directly or indirectly, how pleasant the shampoo bottles looked to the participants, and how congruent a triangular-shaped label looked to the whole packaging.

\section{General Discussion}

In summary, two experiments and one survey study were conducted in order to examine whether the DPTS effect would emerge when the participants had to search for a triangular-shaped label shown on bottles with different designs. The results of the first two experiments consistently revealed that the visual search for a trapezoidal-shaped shampoo bottle with a downward-pointing triangle on its label was faster than when the triangular label on the target bottle pointed upward. These results are consistent with Zhao et al.'s $(2016,2017)$ earlier findings obtained with wine bottles. By contrast, a reversed DPTS trend was actually observed in the accuracy 
data in Experiments 1 and 2, and in the RTs when the data of these two experiments were combined. The results of our final experiment revealed that a downward-pointing triangular-shaped label was perceived to be more incongruent with trapezoidal-shaped bottles than an upward-pointing label. Moreover, our results also suggest that bottle silhouette directly influences the pleasantness ratings, and interacts with the orientation of the triangular label to influence the visual search; whereas cap location only influences subjective ratings by interacting with other design elements such as label orientation or bottle silhouette, but not the visual search.

In the present study, no significant effect of bottle silhouette was found on the overall speed or accuracy of the participants' visual search, whereas the orientation of the triangular-shaped labels did influence the participant's visual search performance. This result appears to be inconsistent with previous findings of global precedence (see Navon, 1977) in visual search, as searching for an orientation oddball was faster when it differed in global orientation than when it differed in line orientation from the distractors (Found \& Müller, 1997; Saarinen, 1994). One possibility here is that bottle contour may not be a global feature, but instead, presenting one element such as a triangular-shaped label on the front of a packaging is more analogous to placing an object within a frame.

We did find that bottle silhouette modulated how triangular-shaped labels influenced visual search, consistent with the orientation search literature that searching for an oriented feature is influenced by the surrounding frame (see Marendaz, 1998, for a review). In laboratory-based studies using simple geometric 
shapes, the emergence of the DPTS effect has been attributed to the threat-related information conveyed by the downward-pointing triangles (Larson et al., 2007, 2009, 2012; Watson et al., 2012). However, when it comes to the usage of downward-pointing triangles in the packaging design for non-threat-related products, such as shampoo bottles (in the present study) or wine bottles (see Zhao et al., 2016, 2017), the results of the present study suggest that the incongruence between the shapes of the label and bottle captures participants' attention more readily. This result is analogous to presenting a target in a surrounding frame which has incongruent shape with it (Bavelier, Deruelle, \& Proksch, 2000; Van Leeuwen \& Lachmann, 2004).

It should be noted that Shen et al. (2015) demonstrate that searching for a downward-pointing triangular-shaped pizza box was faster than for an upward-pointing one. The discrepancy in the pattern of results between that study and the present one suggests that our participants might not consider inverted trapezoidal-shaped and trapezoidal-shaped bottles (as shown in the present study) as downward- and upward-pointing, respectively. While a downward-pointing triangular-shaped label was considered to be more incongruent with the rectangular-shaped bottle with a cap on the bottom in Experiment 3, but we did not find any DPTS or reversed DPTS effect for this bottle in Experiment 1. It is possible that the width difference between the two sides of the trapezoidal-shaped (or inverted-trapezoidal-shaped) bottles is not large enough (i.e., one side was $23.5 \%$ narrower than the other side) for the participants to perceive the narrow side as an 
angular point. However, such wide difference may be substantial enough to elicit perceived incongruence to triangular-shaped labels to guide visual search, thus suggesting the possible difference in the sensitivity of detecting and evaluating

processes to the perceived incongruence between a feature and its reference frame.

In packaging design, bottles are often designed to be comfortable to grasp and hold (Widiyati \& Aoyama, 2013; see also Juravle, Velasco, Salgado-Montejo, \& Spence, 2015), so the bottle silhouette is smooth and round without pointing angles. That being said, the results of the present study do suggest that presenting labels with sharp pointing angles on these smooth and round bottles may be an effective way of capturing shopper's attention in-store or online. Even if placing a downward-pointing triangular shaped label may increase unpleasantness ratings for certain kinds of packaging, such a design may nevertheless help the consumer to differentiate personal care products from food to avoid unintentional self-poisonings (see Basso et al., 2014). Moreover, carefully allocating the flip-top cap may also eliminate the negative influence that a special design in bottle silhouette or label orientation may have caused. Previous research has suggested that consumers prefer a flip-top cap rather than a press pump cap (Sihombing, Jaafar, \& Yuhazri, 2015), presumably because the former allows the consumer to easily sniff the fragrance of the contents prior to purchase (Stalmans, 2008). The result of the present study revealed that a trapezoidal-shaped bottle was perceived to be less pleasant than an inverted-trapezoidal- or rectangle-shaped bottle when having its cap on the top, but 
this effect was eliminated when the cap was moved from the top to the bottom, thus suggesting the importance of cap design.

As with any study, there are certain limitations as far the interpretation and generalizability of the present series of experiments is concerned. For one, the laboratory-based visual search task used in the present study is quite different from how consumers search for a product in real life. In particular, the colours of the packaging and labels were well-controlled in the present study, and only one packaging shape was shown to each participant in each of visual search experiments. It will be interesting to examine any interaction between the colour and shape of the packaging while using heterogeneous distractors having varied shapes and colours. For another, we only used the RTs in a visual search task to assess participants' attention (see Gwinn, Leber, \& Krajbich, 2019 for the logic of doing so), but using eye-tracking technique may be a more straightforward measurement to possibly be incorporated in future studies (Ashby, Walasek, \& Glöckner, 2015; Knoeferle, Knoeferle, Velasco, \& Spence, 2016).

In conclusion, the results of the present study revealed how the appearances of product packaging might guide consumers' visual attention as far as the visual search for shampoo products is concerned. These findings may be of particular importance for the packaging design of beauty products for which the cost of the packaging may even exceed that of the product inside (e.g., Poulter, 2007). Notably, the global cosmetics industry provides valuable business opportunities for those companies delivering products that can be applied to consumers' face, hair, and body (e.g., 
Briney, 2005; Hunt, Fate, \& Dodds, 2011; Tournois, 2013, 2014; Yang, Yang, Xia, \& Sheng, 2012). The findings of the present study may be also of relevance for the packaging of products in other categories, such as foods and beverages (Batra, Seifert, \& Brei, 2015). Even though product packaging is not always related to the functionality of the product (Keller, 1993), it often leads to both sensory and hedonic expectations concerning the products themselves (for a review, see Piqueras-Fiszman \& Spence, 2015), and, as such, can influence consumers' product preference (Barnett, Velasco, \& Spence, 2016; Gatti, Spence, \& Bordegoni, 2014; Piqueras-Fiszman \& Spence, 2012; Stoll et al., 2008; Spence \& Wang, 2017). Skilfully designing bottle silhouette and label shapes to provide contrast or incongruence might be another way to yield twice the result with half the effort, in terms of attracting consumers' attention in-store and increasing the likelihood of products being chosen for purchase. 
TRIANGLES IN PACKAGING DESIGN

\section{Acknowledgments}

This research was supported by the National Natural Science Foundation of China (Grant No. 71472106 \& 71872097) awarded to Xiaoang Wan and and Tsinghua University Initiative Scientific Research Program. The author would like to thank Yihang Ouyang for her technical assistance. Comments concerning this article should be sent to: Dr. Xiaoang Wan at wanxa@mail.tsinghua.edu.cn.

\section{Conflict of Interest}

The authors declare no conflict of interest. 


\section{References}

Arboleda, A. M., \& Arce-Lopera, C. (2015). Quantitative analysis of product categorization in soft drinks using bottle silhouettes. Food Quality \& Preference, $45,1-10$

Ashby, N. J. S., Walasek, L., \& Glöckner, A. (2015). The effect of consumer ratings and attentional allocation on product valuations. Judgment and Decision Making, $10(2), 172-184$.

Barnett, A., Velasco, C., \& Spence, C. (2016). Bottled vs. canned beer: Do they really taste different? Beverages, 2:25.

Basso, F., Robert-Demontrond, P., Hayek, M., Anton, J., Nazarian, B., \& Roth, M. (2014). Why people drink shampoo? Food imitating products are fooling brains and endangering consumers for marketing purposes. PLoS ONE 9(9):e100368.

Batra, R., Seifert, C., \& Brei, D. (2015). The psychology of design: Creating consumer appeal. London, UK: Routledge.

Bavelier, D., Deruelle, C., \& Proksch, J. (2000). Positive and negative compatibility effect. Perception \& Psychophysics, 62, 100-112.

Briney, C. (2005). Industry growth on the horizon. Global Cosmetic Industry, 173, 41-42.

Chitturi, R., Raghunathan, R., \& Mahajan, V. (2008). Delight by design: The role of hedonic versus utilitarian benefits. Journal of Marketing, 72(3), 48-63.

Clement, J., Kristensen, T., \& Grønhaug, K. (2013). Understanding consumers' in-store visual perception: The influence of package design features on visual 
attention. Journal of Retailing and Consumer Services, 20(2), 234-239.

Creusen, M. E., \& Schoormans, J. P. (2005). The different roles of product appearance in consumer choice. Journal of Product Innovation Management, 22(1), 63-81.

Found, A., \& Müller, H. J. (1997). Local and global orientation in visual search. Perception \& Psychophysics, 59, 941-963.

Gatti, E., Spence, C., \& Bordegoni, M. (2014). Investigating the influence of colour, weight, \& fragrance intensity on the perception of liquid bath soap. Food Quality \& Preference, 31, 56-64.

Grundey, D. (2010). Functionality of product packaging: Surveying consumers' attitude towards selected cosmetic brands. Economics \& Sociology, 31(1), 87-103.

Gwinn, R., Leber, A. B., \& Krajbich, I. (2019). The spillover effects of attentional learning on value-based choice. Cognition, 182, 294-306.

Hunt, K. A., Fate, J., \& Dodds, B. (2011). Cultural and social influences on the perception of beauty: A case analysis of the cosmetic industry. Journal of Business Case Studies, 7(1), 1-10.

Juravle, G., Velasco, C., Salgado-Montejo, A., \& Spence, C. (2015). The hand grasps the centre, while the eyes saccade to the top of novel objects. Frontiers in Psychology, 6:633.

Keller, K. L. (1993). Conceptualizing, measure, and managing customer-based brand equity. Journal of Marketing, 57, 1-22.

Knoeferle, K., Knoeferle, P., Velasco, C., \& Spence, C. (2016). Multisensory brand 
search: How the meaning of sounds guides consumers' visual attention. Journal of Experimental Psychology: Applied, 22, 196-210.

Kunde, W., \& Hoffman, J. (2000). Global-local orientation congruency effects in visual search. Quarterly Journal of Experimental Psychology, 53A, 537-548.

Larson, C. L., Aronoff, J., Sarinopoulos, I. C., \& Zhu, D. C. (2009). Recognizing threat: Simple geometric shapes activate neural circuitry underlying threat detection. Journal of Cognitive Neuroscience, 21, 1523-1535.

Larson, C. L., Aronoff, J., \& Stearns, J. J. (2007). The shape of threat: Simple geometric forms evoke rapid and sustained capture of attention. Emotion, 7, $526-534$.

Larson, C. L., Aronoff, J., \& Steuer, E. L. (2012). Simple geometric shapes are implicitly associated with affective value. Motivation and Emotion, 36, 404-413.

Marendaz, C. (1998). Nature and dynamics of reference frames in visual search for orientation: Implications for early visual processing. Psychological Science, 9(1), $27-32$

May, K. A., \& Li, Z. (2009). Effects of surrounding frame on visual search for vertical or tilted bars. Journal of Vision, 9(13):20.

Navon, D. (1977). Forest before trees: The precedence of global features in visual perception. Cognitive Psychology, 9(2), 353-383.

Orquin, J. L., \& Loose, S. M. (2013). Attention and choice: A review on eye movements in decision making. Acta Psychologica, 144(1), 190-206.

Orth, U. R., \& Malkewitz, K. (2008). Holistic package design and consumer brand 
impressions. Journal of Marketing, 72, 64-81.

Pieters, R., \& Warlop, L. (1999). Visual attention during brand choice: The impact of time pressure and task motivation. International Journal of Research in Marketing, 16(1), 1-16.

Pieters, R., Warlop, L., \& Wedel, M. (2002). Breaking through the clutter: Benefits of advertisement originality and familiarity for brand attention and memory. Management Science, 48(6), 765-781.

Pieters, R., Wedel, M., \& Batra, R. (2010). The stopping power of advertising: Measures and effects of visual complexity. Journal of Marketing, 74, 48-60.

Piqueras-Fiszman, B., \& Spence, C. (2012). The weight of the bottle as a possible extrinsic cue with which to estimate the price (and quality) of the wine? Observed correlations. Food Quality \& Preference, 25, 41-45.

Piqueras-Fiszman, B., \& Spence, C. (2015). Sensory expectation based on product-extrinsic food cues: An interdisciplinary review of the empirical evidence and theoretical accounts. Food Quality and Preference, 40, 165-179.

Poulter, S. (2007). Product packaging can cost three times as much as what's inside. Retrieved online from http://www.dailymail.co.uk/news/article-468022 on August 9th, 2018.

Saarinen, J. (1994). Visual search for global and local stimulus features. Perception, $23,237-243$.

Salgado-Montejo, A., Salgado, C., Alvarado, J., \& Spence, C. (2017). Simple lines and shapes are associated with, and communicate, distinct emotions. 
TRIANGLES IN PACKAGING DESIGN

Cognition \& Emotion, 31, 511-525.

Selame, T., and Koukos, P. (2002). Is your package shelf-evident? Design Management Journal, 13(4), 25-31.

Shen, X., Wan, X., Mu, B., \& Spence, C. (2015). Searching for triangles: An extension to food \& packaging. Food Quality and Preference, 44, 26-35.

Sihombing, H., Jaafar, R., \& Yuhazri, M. Y. (2015). The satisfaction preferences measure of profile design. Applied Mechanics and Materials, 815, 304-312.

Simmonds, G., \& Spence, C. (2017). Thinking inside the box: How seeing products on, or though, the packaging influences consumer perceptions and purchase behavior. Food Quality and Preference, 62, 340-351.

Spence, C. (2016). Multisensory packaging design: Color, shape, texture, sound, and smell. In P. Burgess (Ed.), Integrating the packaging and product experience: A road-map to consumer satisfaction (pp. 1-22). Oxford, UK: Elsevier.

Spence, C., \& Wang, (Q.) J. (2017). Assessing the impact of closure type on wine ratings and mood. Beverages, 3:52.

Stalmans, M. (2008). The story behind fresh fragrances: The science and safety of P\&G's fabric and air freshener perfumes. Household and Personal Care Today, 3, 50-54.

Stoll, M., Baecke, S., \& Kenning, P. (2008). What they see is what they get? An fMRI study on neural correlates of attractive packaging. Journal of Consumer Behaviour, 7, 342-359.

Tournois, L. (2013). Mass market leadership and shampoo wars: The L'Oreal strategy. 
TRIANGLES IN PACKAGING DESIGN

Journal of Business Strategy, 34(1), 4-14.

Tournois, L. (2014). Too many products? Reaching the next billion customers of the beauty market. Journal of Business Strategy, 35(5), 3-13.

Towal, R. B., Mormann, M., \& Koch, C. (2013). Simultaneous modeling of visual saliency and value computation improves predictions of economic choice. Proceedings of the National Academy of Sciences of the United States of America, 110(40), E3858-E3867.

Van Leeuwen, C., \& Lachmann, T. (2004). Negative and positive congruence effects in letters and shapes. Perception \& Psychophysics, 66(6), 908-925.

Velasco, C., Woods, A. T., \& Spence, C. (2015). Evaluating the orientation of design elements in product packaging using an online orientation task. Food Quality \& Preference, 46, 151-159.

Wang, E. (2013). The influence of visual packaging design on perceived food product quality, value, and brand preference. International Journal of Retail \& Distribution Management, 41(10), 805-816.

Watson, D. G., Blagrove, E., Evans, C., \& Moore, L. (2012). Negative triangles: Simple geometric shapes convey emotional valence. Emotion, 12, 18-22.

Westerman, S. J., Sutherland, E. J., Gardner, P. H., Baig, N., Critchley, C., \& Hickey, C. et al. (2013). The design of consumer packaging: Effects of manipulations of shape, orientation, and alignment of graphical forms on consumers' assessments. Food Quality and Preference, 27(1), 8-17. 
Widiyati, K., \& Aoyama, H. (2013). The ease of grasping to evaluate aesthetically pleasing pet bottle design. Journal of Advanced Mechanical Design, Systems, and Manufacturing, 7(5), 849-861.

Yang, C., Yang, F., Xia, Q., \& Sheng, A. (2012). What makes sales in Chinese shampoo industry? A DEA study based on efficient market hypothesis. Asia Pacific Journal of Marketing and Logistics, 24(4), 678-689.

Zaichkowsky, J. L. (2010). Strategies for distinctive brands. Brand Management, $17(8), 548-560$.

Zbib, I. F., Wooldridge, B. R., Ahmed, Z. U., \& Benlian, Y. (2010). Purchase of global shampoo brands and the impact of country of origin on Lebanese consumers. Journal of Product \& Brand Management, 19, 261-275.

Zhao, H., Huang, F., Spence, C., \& Wan, X. (2017). Visual search for wines with a triangle on the label in a virtual store. Frontiers in Psychology, 8, 2173-2083.

Zhao, H., Spence, C., \& Wan, X. (2016). Visual search for triangles in wine labels. Proceedings of the 1st Workshop on Multi-sensorial Approaches to Human-Food Interaction, Article No. 3. November 16, Tokyo, Japan. 
Figures
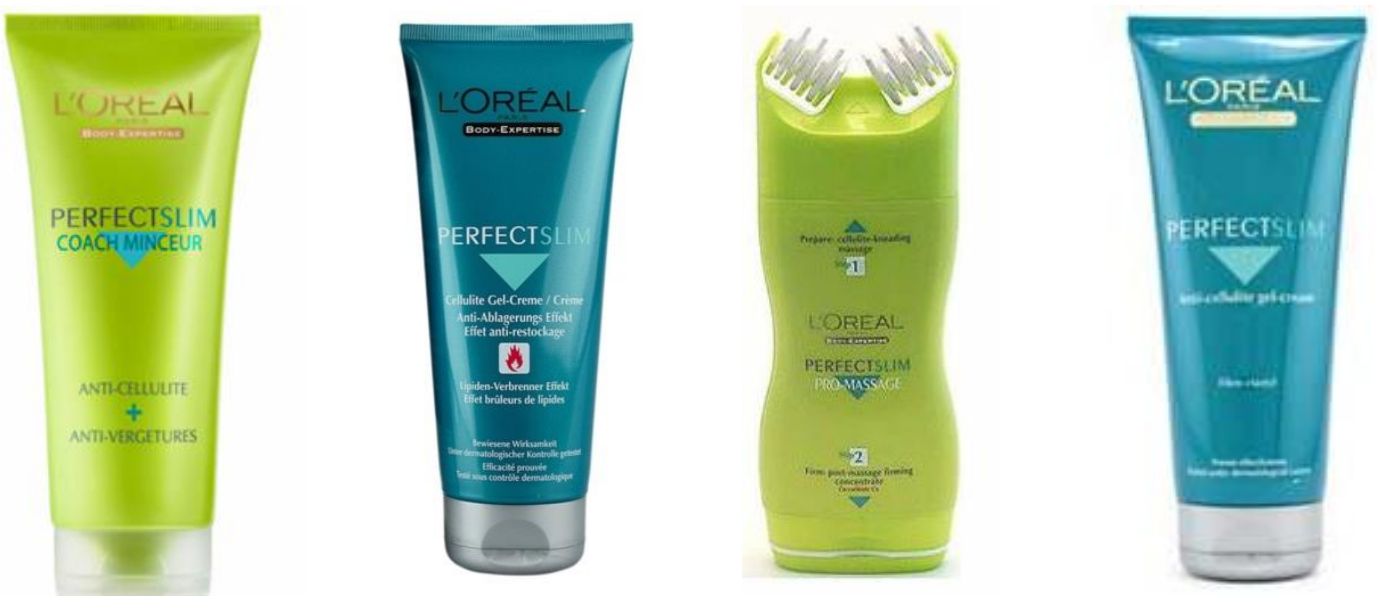

Figure 1. Commercial examples of downward-pointing triangles used in the packaging of skin care products. 
Bottle silhouettes

Inverted-trapezoidal shaped

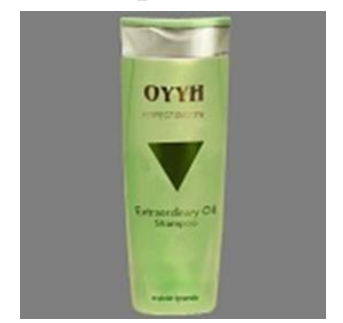

Trapezoidal-shaped

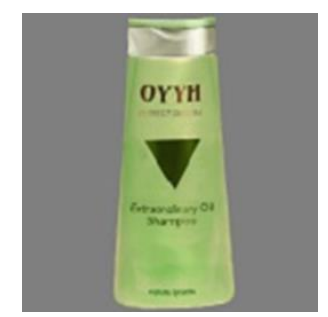

Rectangular-shaped

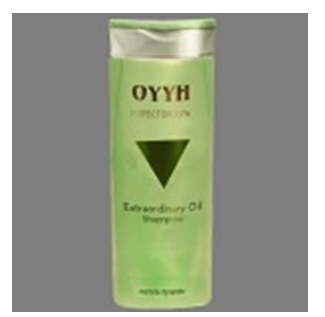

Figure 2. Sample illustrations of three types of bottle silhouettes used in the present study, including an inverted-trapezoidal shape in which the bottom was $23.5 \%$ narrower than the top, a trapezoidal shape in which the top was $23.5 \%$ narrower than the bottom, and a rectangular shape in which the top and bottom were of the same widths. 

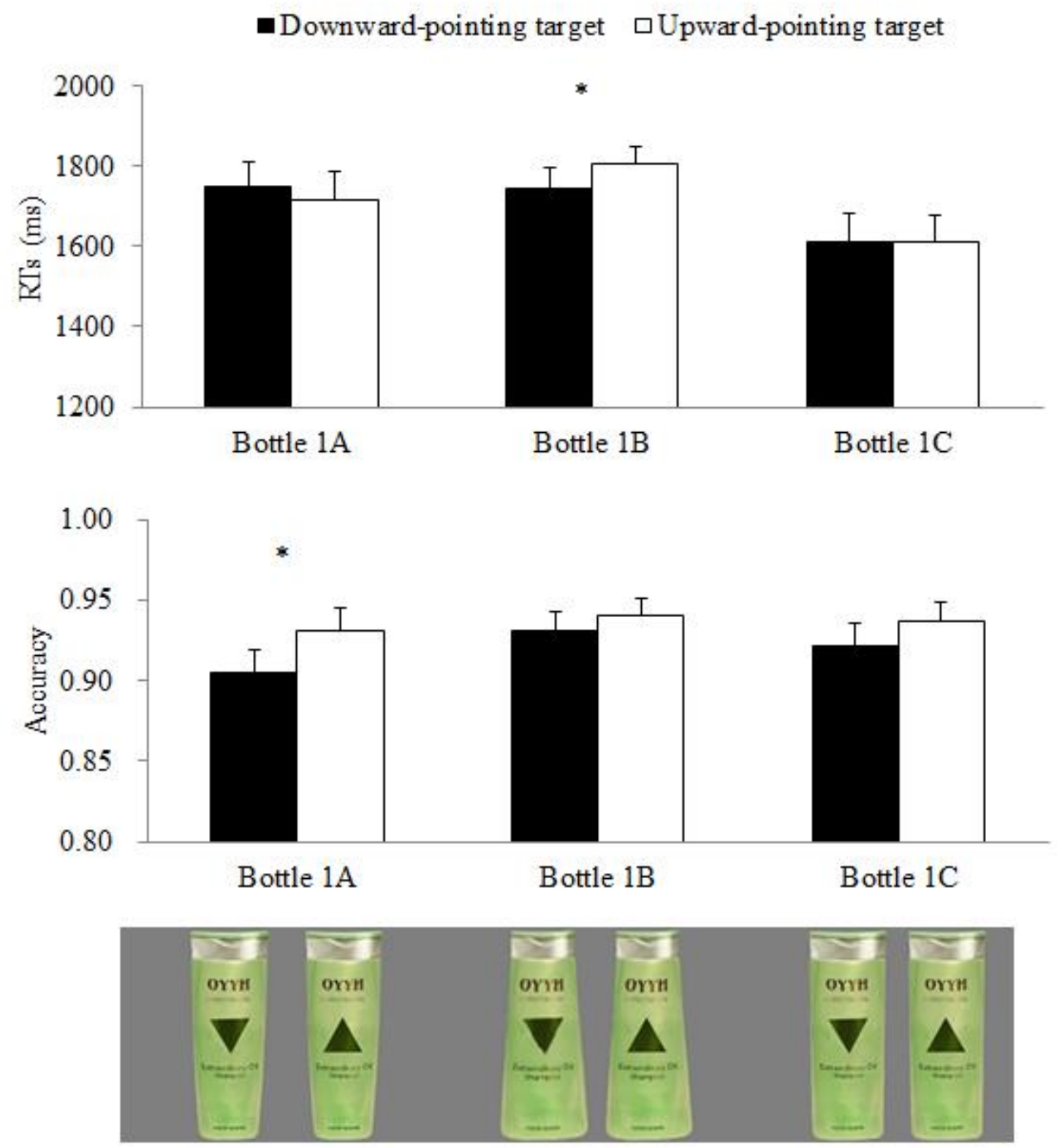

Figure 3. Mean RTs and accuracy in each condition for different types of bottles in Experiment 1 . Note $*$ denotes $p<0.05$, and the error bars show the standard errors of the means. 

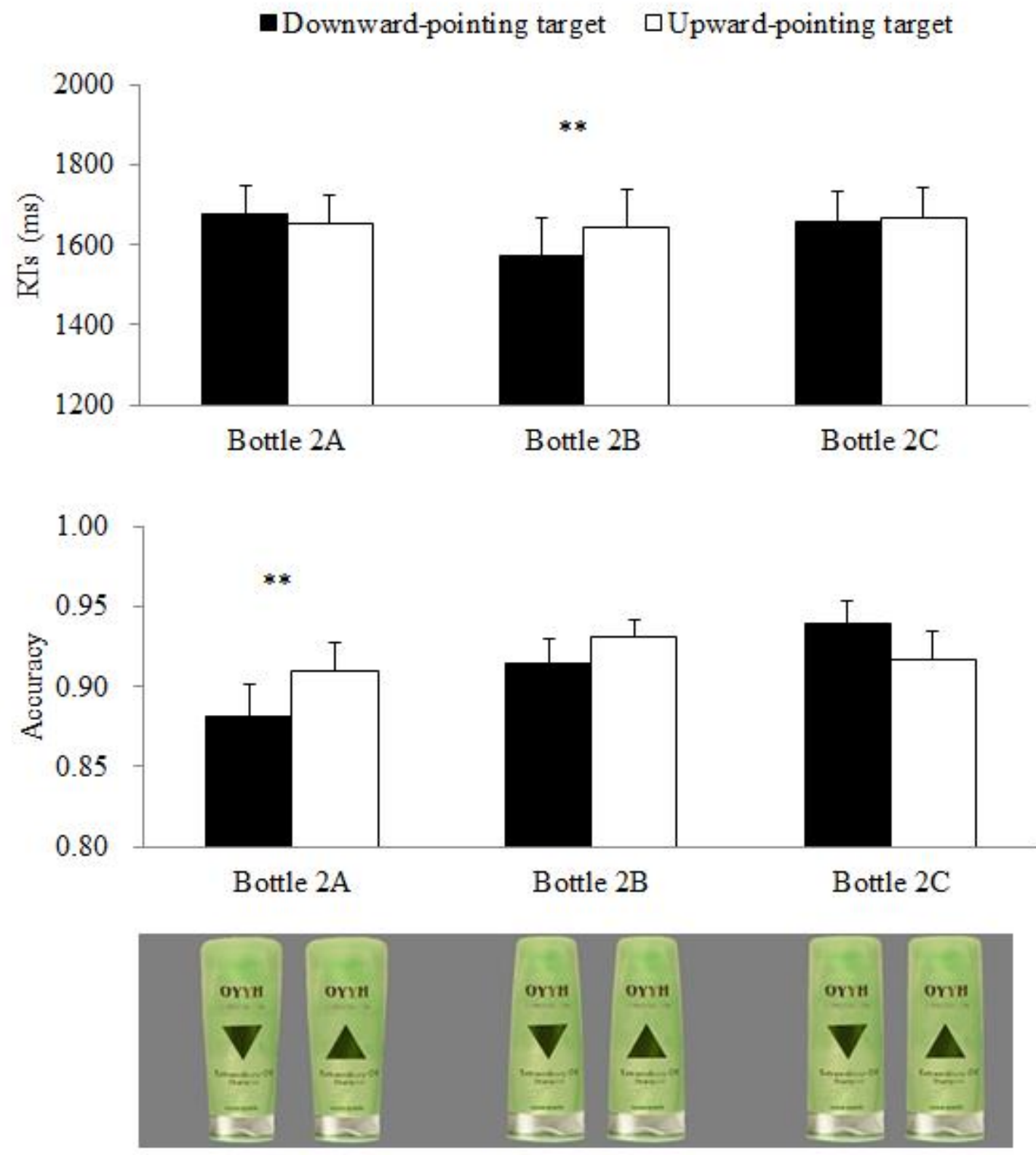

Figure 4. Mean RTs and accuracy in each condition for different types of bottles in Experiment 2. Note $* *$ denotes $p<0.01$, and error bars show the standard errors of the means. 

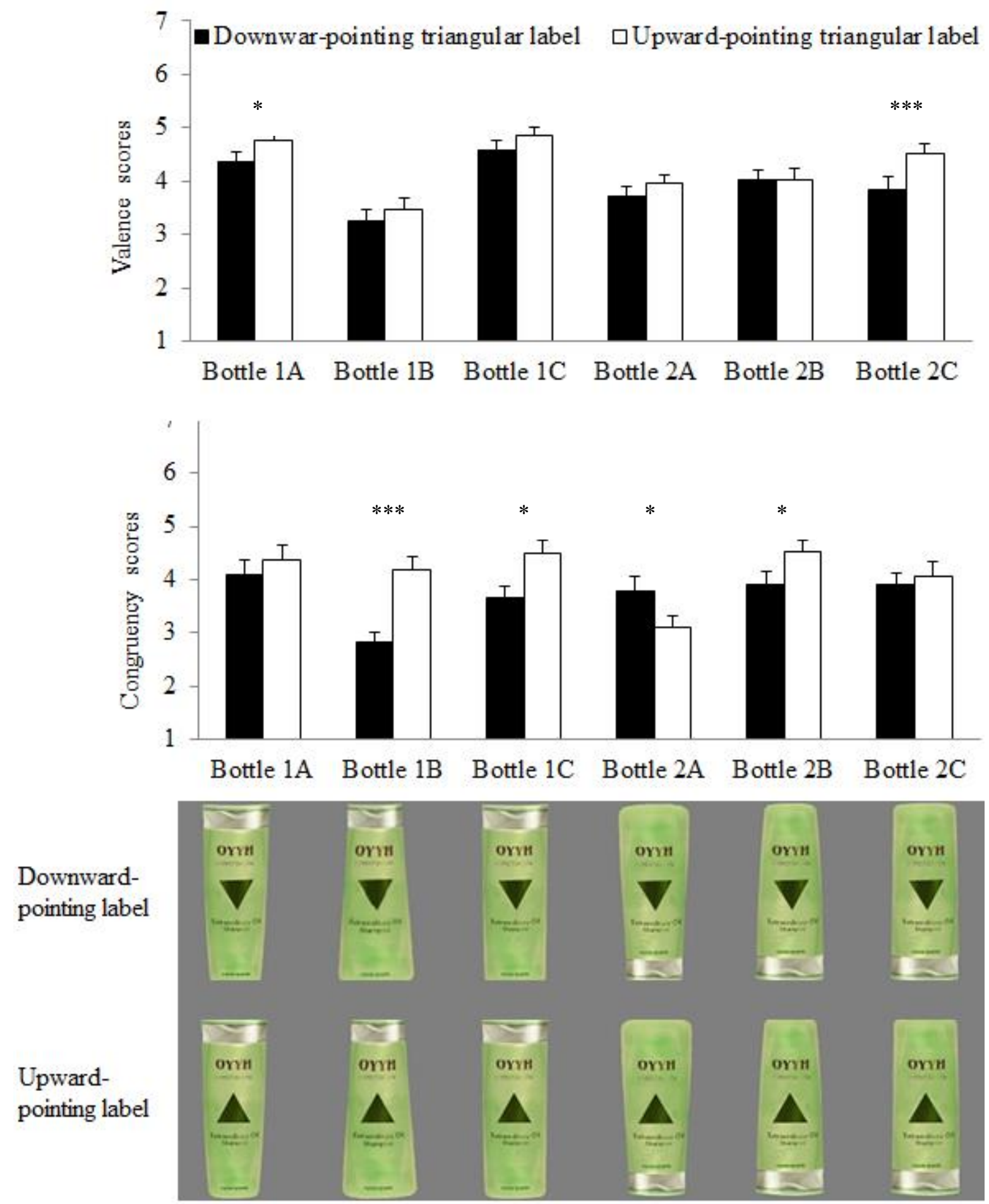

Figure 5. The mean ratings of valence and congruence for different bottles tested in Experiment 3. Error bars showed the standard errors of the means. Note $*$ denotes $p<$ 0.05 , and $* * *$ denotes $p<0.001$. 